\title{
Fishes of Caxiuanã National Forest: 20 years (1993 to 2012) of sampling in a protected area in the Eastern Amazon
}

\section{Peixes da Floresta Nacional de Caxiuanã: 20 anos (1993 a 2012) de amostragem em uma área protegida da Amazônia Oriental}

\author{
Tiago Magalhães da Silva Freitas', Bruno da Silveira Prudente", Danielly Torres Hashiguti de Freitas"II, \\ Naraiana Loureiro Benone ${ }^{\mathrm{IV}}$, Híngara Leão ${ }^{\mathrm{IV}}$, Guilherme Moreira Dutra ${ }^{\mathrm{V}}$, Luciano Fogaça de Assis Montag ${ }^{\mathrm{IV}}$ \\ Universidade Federal do Pará. Campus Universitário do Marajó. Breves, Pará, Brasil \\ "Universidade Federal Rural da Amazônia. Capitão Poço, Pará, Brasil \\ I'U Universidade da Amazônia. Belém, Pará, Brasil \\ vUniversidade Federal do Pará. Belém, Pará, Brasil \\ vMuseu Paraense Emílio Goeldi/MCTIC. Programa de Capacitação Institucional. Belém, Pará, Brasil
}

\begin{abstract}
The Amazon basin comprises the most diverse freshwater fish fauna in the world, but remains poorly-known concerning the potential number of species. This study aims to produce an updated checklist of fish species from the Caxiuanã National Forest (FLONA Caxiuanã), a protected area of the Eastern Amazon. Sampling from 20 years (1993 to 2012) was compiled from the ichthyological collection of the Museu Paraense Emilio Goeldi and previously published inventories. A total of 211 fish species belonging to 44 families and 13 orders was recognized for the FLONA Caxiuanã. Characiformes was the richest order with 80 species (representing 38\% of the total), followed by Siluriformes (56 species, 27\%), Perciformes (33 species, 16\%), and Gymnotiformes (21 species, 10\%). Almost 40\% of the fish diversity was considered new records for the Caxiuanã region, with a higher species increment occurring in 2004, and a lower average increment of species in the last years. Therefore, additional species records could be obtained if more effort were applied in surveying the ichthyofaunal, considering different fishing techniques, and covering different microhabitats.
\end{abstract}

Keywords: Ichthyofauna. Inventory. Amazon basin.

Resumo: A bacia amazônica possui a maior diversidade de peixes de água doce do mundo, mas ainda é insatisfatoriamente conhecida quanto ao número real de espécies. Este estudo objetiva produzir uma lista atualizada de espécies de peixes da Floresta Nacional de Caxiuanã (FLONA Caxiuanã), uma das poucas áreas protegidas da Amazônia Oriental. Dados referentes a 20 anos (1993 a 2012) de amostragem foram compilados da coleção ictiológica do Museu Paraense Emílio Goeldi e de inventários previamente publicados. Um total de 211 espécies de peixes pertencentes a 44 famílias e a 13 ordens foi identificado para a FLONA. Characiformes foi a ordem mais rica, com 80 espécies (representando 38\% do total), seguido por Siluriformes (56 espécies, 27\%), Perciformes (33 espécies, 16\%) e Gymnotiformes (21 espécies, 10\%). Do total da diversidade de peixes registrada, quase $40 \%$ representam novos registros, com maior incremento de espécies no ano de 2004 e menor incremento médio de espécies nos últimos anos de amostragem. Portanto, registros de espécies adicionais ainda podem ser obtidos se estudos na região relacionados à ictiofauna empregarem diferentes técnicas e apetrechos de coleta e forem realizados em diferentes microhabitats.

Palavras-chave: Ictiofauna. Inventário. Bacia amazônica.

FREITAS, T. M. S., B. S. PRUDENTE, D. T. H. FREITAS, N. L. BENONE, H. LEÃO, G. M. DUTRA \& L. F. A. MONTAG, 2018. Fishes of Caxiuanã: 20 years (1993 to 2012) of sampling in a protected area in the Eastern Amazon. Boletim do Museu Paraense Emílio Goeldi. Ciências Naturais 13(2): 185-204.

Autor para correspondência: Tiago Magalhães da Silva Freitas. Universidade Federal do Pará. Campus Universitário do Marajó-Breves. Faculdade de Ciências Naturais. Alameda IV, 3418 - Parque Universitário. Breves, PA, Brasil. CEP 68800-000 (freitastms@gmail.com). Recebido em 07/08/2017

Aprovado em 18/03/2018

Responsabilidade editorial: Fernando da Silva Carvalho Filho

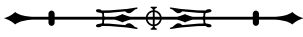




\section{INTRODUCTION}

The freshwater fish fauna of South America is the most diverse on Earth, with recent estimates standing between 8,000 and 9,000 species and currently more than 5,600 species formally described (Reis et al., 2016). Within South America, the Amazon basin, the largest and most diverse freshwater basin in the world, contains an expressive part of this total, with more than 2,400 species described (Reis et al., 2003, 2016).

Gaining a better knowledge of this fish diversity depends on more effort devoted to inventories (Ota et al., 2015), although detecting and describing the fauna of a certain region, and interpreting the data obtained in the field, is not an easy task. Generally, in megadiverse groups such as fish, taxonomic knowledge is changeable due to the description of new species, taxonomic revisions, formation of new specialists, and different sampling effort in different aquatic environments (Silveira et al., 2010; Ota et al., 2015; Hurd et al., 2016). On the other hand, direct anthropogenic activities such as deforestation, pollution, demographic occupation, reservoir construction, and overexploitation, contribute to the decline of fish diversity (Junk et al., 2007; Winemiller et al., 2016), including the extinction of species that have not even been described. Thus, the establishment of protected areas is an important strategy for the maintenance of natural environments and genetic patrimony, and the conservation of biodiversity (Silva-Oliveira et al., 2016). Since the 1980s, a network of protected areas has been created in the Amazon as a response to pressing environmental issues regarding forests and their biodiversity, but little attention has been paid to managing freshwater ecosystems (Castello et al., 2013).

The Caxiuanã National Forest (hereafter FLONA Caxiuanã), a federal sustainable use protected area located in Pará State, was created in November 1961, in accordance with the Brazilian system of protected areas (Brasil, 2011), and is the second oldest protected area in Brazil. In 1993, in the FLONA Caxiuanã, the Ferreira Penna Field Station (in Brazilian Portuguese: Estação Científica Ferreira Penna) was installed.
The station is managed by the Museu Paraense Emílio Goeldi and the Ministério de Ciência, Tecnologia, Inovações e Comunicações, which conduct research, mainly of long duration, on the local biodiversity (Lisboa et al., 2013).

The first checklist of fishes from FLONA Caxiuanã was based on sporadic samples, resulting in 41 species with no voucher specimens being deposited in a scientific collection (Lisboa, 1997). The second checklist comprised 208 fish species that were cataloged from samples collected between 1999 and 2004 (Montag et al., 2008), sampling mainly the open waters of rivers and bays.

Thereafter, many ecological studies of fish population of FLONA Caxiuanã have been carried out, focused on most representative species (Freitas et al., 2011a, 2011b, 2014, 2016, 2017; Prudente et al., 2015, 2016; Hashiguti et al., 2017; Oliveira et al., 2017), which occasionally resulted only in new records of species. Studies of stream fish assemblages conducted by Sousa (2014) and Benone et al. (2018) also have contributed with new records of species for Caxiuanã, since they have extensively investigated a poorly-sampled environment, the stream ecosystem.

Thus, considering the above-mentioned studies and their large number of samplings carried out after Montag et al. (2008), and the advances in freshwater fish taxonomy, the aim of this study was to produce an updated checklist of fish species of the FLONA Caxiuanã.

\section{MATERIALS AND METHODS}

The Caxiuanã National Forest in Eastern Amazonia, state of Pará, Brazil (between $1^{\circ} 37^{\prime}$ S, $51^{\circ} 19^{\prime} \mathrm{W}$ and $2^{\circ} 15^{\prime} \mathrm{S}$, $51^{\circ} 55^{\prime}$ W) (Figure 1) holds an area over 300,000 hectares encompassing part of Portel and Melgaço municipalities (Lisboa et al., 2013). The FLONA Caxiuanã is part of the Xingu and Tocantins rivers systems in a lowland Amazon plain. Owing to a geological event leading to the drowning of valleys of the Anapu River during the Holocene (Behling \& Costa, 2000), which define the region as a 'ria lake' that show little variation in fluviometry between hydrological periods (Costa et al., 2002). 


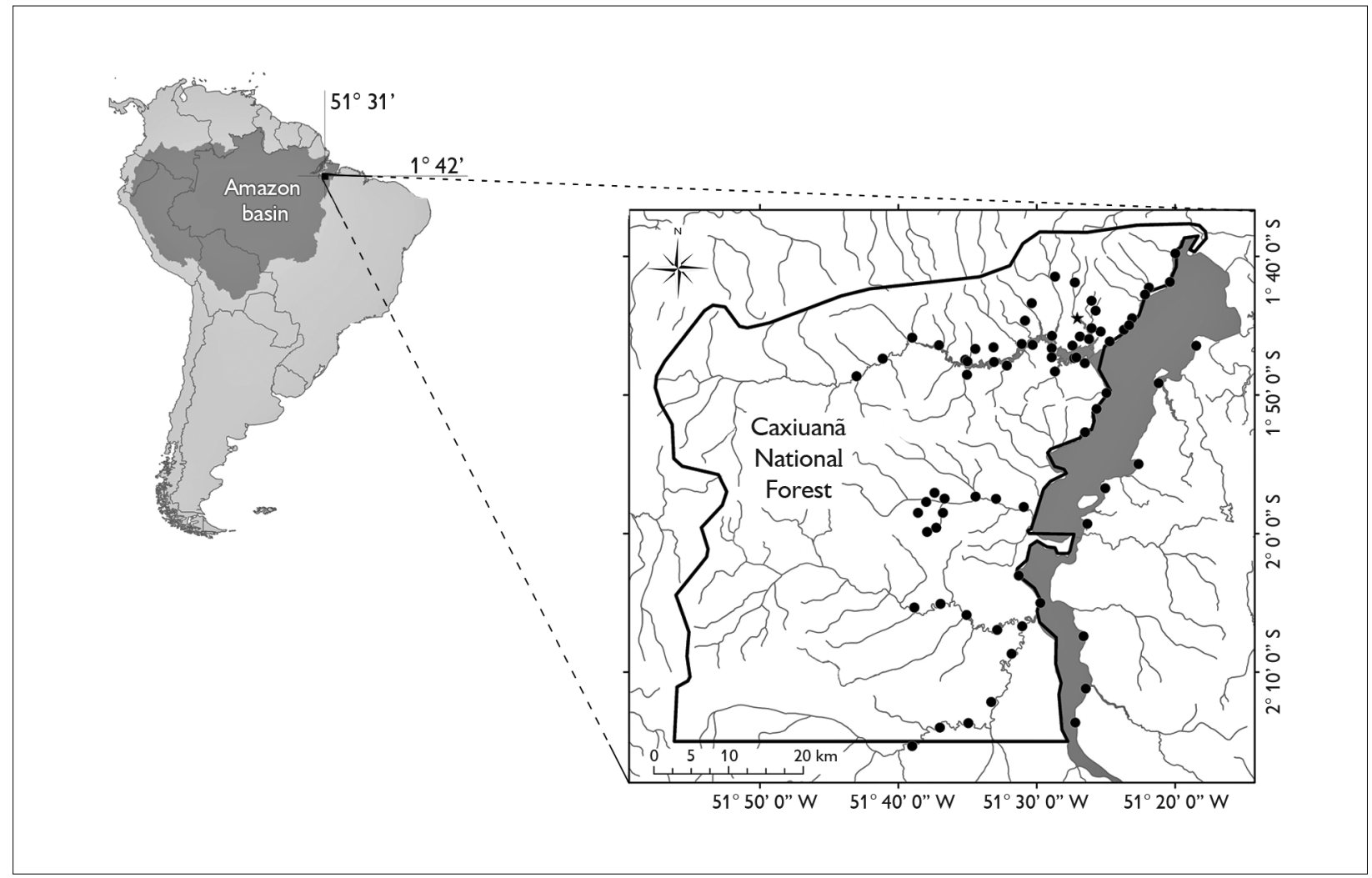

Figure 1. Location of the Caxiuanã National Forest, showing the sites where fish sampling occurred (period 1993 to 2012). The star symbol represents the Scientific Station (Estação Científica Ferreira Penna).

The updated checklist was based on vouchers specimens collected in FLONA Caxiuanã and catalogued in the fish collection of the Museu Paranse Emilio Goeldi (MPEG). All species had their vouchers revised in part, according to recent taxonomic revisions, and studies without voucher specimens were not considered herein. Current nomenclature follows the Catalog of Fishes (Eschmeyer et al., 2017).

According to the local where a species was registered, they were assigned to different aquatic environments (river/bay, stream, floating bank); more detailed environmental descriptions can be found in Montag et al. $(2008,2013)$. The fish species were also checked for their potential exploitation by the ornamental fishery, based on the list of ornamental fish species (Brasil, 2012), and for their threat status considering the list of threatened species of Pará (Pará, 2007), the Brazilian list of threatened species (Brasil, 2014a), and the IUCN Red List of Threatened Species (IUCN, 2017). Finally, an accumulation curve over the 20 years of non-standardized fish samplings (1993 to 2012) was performed based in the year of first record for each species.

\section{RESULTS}

A total of 211 fish species belonging to 44 families and 13 orders were recognized for the FLONA Caxiuanã (see Appendix 1 for checklist and Appendix 2 for voucher codes). Characiformes was the richest order with 80 species (38\% of total), followed by Siluriformes (56 spp., 27\%), Perciformes (33 spp., 16\%), and Gymnotiformes (21 spp., 10\%). The other nine orders represented $9.5 \%$ (20 spp.) of total species richness. 
The most representative families in number of species were Cichlidae (28 spp., 13.3\%), followed by Characidae (22 spp., 10.5\%), Auchenipteridae (15 spp., 7.1\%), Loricariidae and Serrasalmidae (12 spp., 5.7\% each), and Pimelodidae and Lebiasinidae (10 spp., 4.7\% each) (Figure 2).

Most species (110 spp., 52.1\% of total) were recorded only in rivers and bays, followed by species recorded in streams (44 spp., 20.9\%) and species in banks of floating macrophytes (11 spp., 5.2\%). A total of 14 species were found both in rivers/bays and streams (6.6\%); seven species occurred both in rivers/bays and in floating macrophytes (3.3\%); and 14 species were commonly registered in streams and floating macrophytes (6.6\%). Eight species (3.8\%) were registered for the three environments: Crenuchus spilurus (Crenuchidae), Hoplias malabaricus (Erythrinidae), Megalechis picta, Megalechis thoracata (Callichthyidae), Acaronia nassa, Mesonauta festivus (Cichlidae), Colomesus asellus (Tetraodontidae), and Synbranchus marmoratus (Synbranchidae). Three species (1.4\%) had no habitat information.

Overall, 91 species (31 fish families) can be exploited for ornamental purposes, which represents $43.1 \%$ of the total diversity. Most of them belongs to the families Cichlidae (18 spp.) and Characidae (15 spp.). None species in this study is listed as threatened.

Regarding the assessment of species record accumulation, the species recorded in FLONA Caxiuanã in the three first sampled years (1993, 1999, and 2000) represented $46 \%$ (98 spp.) of the total fish diversity. The following years (2001, 2002, and 2003) registered an increment of 2, 19, and 17 species, respectively. The highest species additions occurred in 2004 with 58 new records, reaching $91.9 \%$ of the cumulative species richness (Figure 3). In the last years of sampling (2005 to 2012), two species were added as an average per year, with the greatest contribution in 2010 (6 spp.) due to sampling in stream ecosystems.

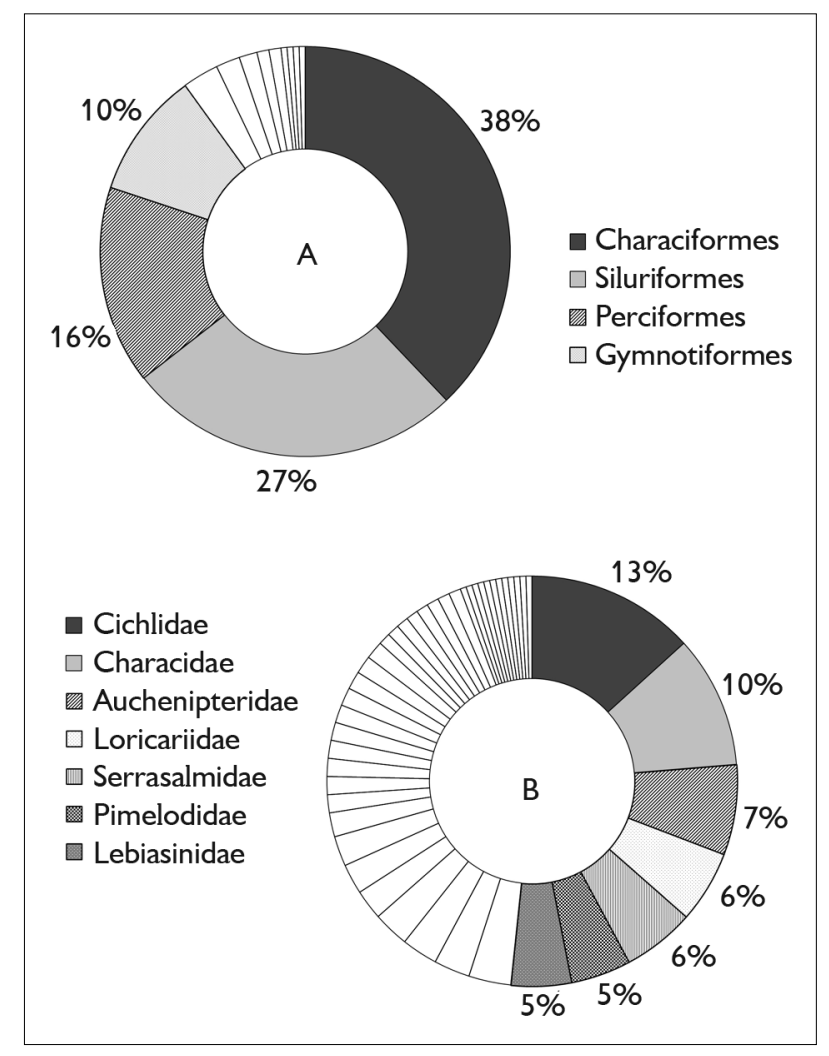

Figure 2. Representativeness of fish species by orders (A) and most diverse families (B) in the Caxiuanã National Forest, Eastern Amazon, Brazil. Empty sections accounted less than $10 \%$ and $5 \%$ of species richness (orders and family, respectively).

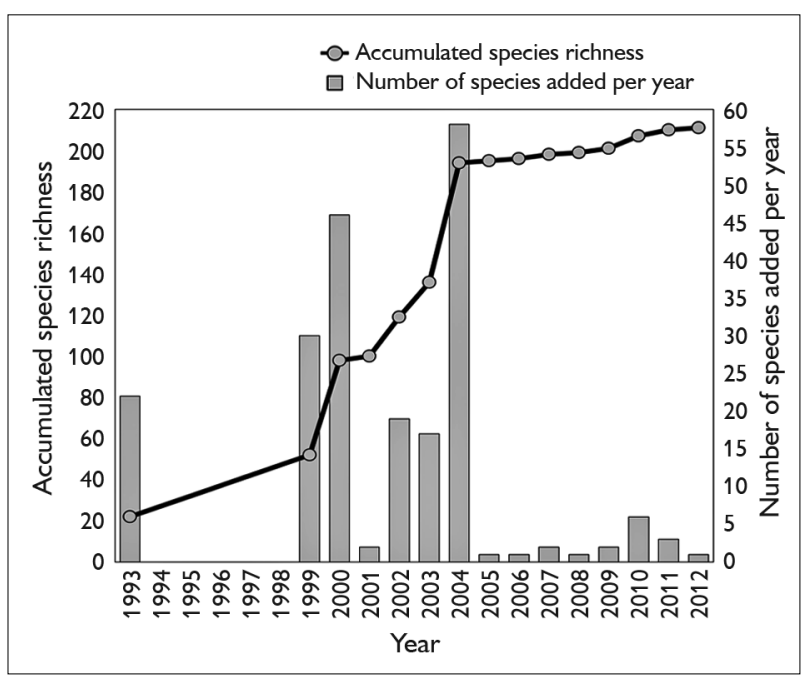

Figure 3. Species accumulation curve and new records of fish species sampled between 1993 to 2012 in the Caxiuanã National Forest, Eastern Amazon, Brazil. 


\section{DISCUSSION}

In the Neotropical region, the fish diversity is mainly composed of Characiformes, followed by Siluriformes, and Gymnotiformes or Perciformes (Lowe-McConnell, 1999; Albert et al., 2011; Claro-García et al., 2013; Melo et al., 2016; Silva-Oliveira et al., 2016). In the present study, Perciformes was the third richest order. The higher diversity of this order, when compared with Gymnotiformes, may be attributed to the predominance of Cichlidae (Perciformes) in slow-flowing rivers (Kullander, 2003), as noticed in the 'ria lake' system of FLONA Caxiuanã (Behling \& Costa, 2000). Additionally, the lower richness of Gymnotiformes may be attributed to less sampling effort in environments such as deep-channel rivers, which are commonly occupied by Gymnotiformes species (Cox-Fernandes et al., 2004).

Within the Neotropical fish fauna, Characidae, Loricariidae, and Cichlidae are the families with the greatest number of valid species (Reis et al., 2016), which reflects the predominance of these families in previous inventory studies. In the present study, Cichlidae was the most diverse fish family, which can be attributed by the predominance of the slow-flow habitats, as mentioned above. The inversion in the number of species between Cichlidae and Loricariidae was also reported in some studies (e.g. Barros et al., 2011; Melo et al., 2016; SilvaOliveira et al., 2016).

Despite the increment of only three species to the total fish diversity in FLONA Caxiuanã since Montag et al. (2008), who reported 208 species versus 211 species in our study, the present list reports 79 new records of species to the area ( $40 \%$ of total). A sizable part of this increment was due to checking taxonomic updates of vouchers (50 spp.), which also reduced identification inaccuracies. Samplings that occurred between 2005 and 2012 also registered new records of species for the FLONA Caxiuanã (29 spp.), mostly because of greater sampling effort in $1^{\text {st }}$ to $3^{\text {rd }}$ order streams (Sousa, 2014; Benone et al., 2018).
Recently, four species were described from material collected in the FLONA Caxiuanã, namely: Physopyxis ananas, Hypopygus benoneae, Eigenmannia antonioi, and Tatia caxiuanensis. The last three species are currently known only from the Anapu River drainage at FLONA Caxiuanã (Sarmento-Soares \& Martins-Pinheiro, 2008; Peixoto et al., 2013, 2015). Nevertheless, at least five new records (e.g., Hyphessobrycon sp., Hypoptopoma sp., Peckoltia sp., Pimelodus sp., and Priocharax sp.) probably correspond to undescribed species, showing that the local ichthyofauna remains not fully-known.

Considering that the locality is a protected area that aims at the sustainable exploitation of natural resources, more than $40 \%$ of recorded species are considered for ornamental fishing (e.g., cichlids, characids, and lebiasinids). A managed exploitation of these species, after population dynamics studies, may comprise an important economic activity for the region. Additionally, no species is considered endangered in the list of threatened species of the state of Pará (Pará, 2007), in the Brazilian list of threatened species (Brasil, 2014a), nor in the IUCN Red List of Threatened Species (IUCN, 2017). All these data should be carefully revisited due to the recently approved environmental licensing for reduced-impact logging in almost 200,000 hectares for the next 40 years within FLONA Caxiuanã (ICMBIO, 2013; Brasil, 2014b). Despite being considered a sustainable practice with low influence on Amazonian fish assemblages (Dias et al., 2010), this activity can modify the stream physical habitat structure (Prudente et al., 2017), compromising fish diversity, especially for the endemic species.

Finally, additional species records could be obtained by devoting more effort to surveying the ichthyofauna by applying diverse fisheries techniques (e.g., trawl nets) and covering different microhabitats. Such efforts are likely to increase the number of species records, particularly if employed in the western region of the FLONA Caxiuanã. This effort can contribute to a reduction of biogeographical and ecological shortfalls in fish faunas and biodiversity conservation. 


\section{ACKNOWLEDGEMENTS}

The authors are grateful to each person who participated in field expeditions, especially the field assistants Benedito Brazão and Renato Calisto. We also thank the institutions that, during this period (1999 to 2012), financed the ichthyological research in FLONA Caxiuanã: Coordenação de Aperfeiçoamento de Pessoal de Nível Superior (CAPES), World Wildlife Found (WWF-BR), Fundo Nacional do Meio Ambiente (FNMA), Fundação Grupo O Boticário de Proteção à Natureza, Programa de Pesquisa em Biodiversidade (PPBio/ Caxiuanã), and Conselho Nacional de Desenvolvimento Científico e Tecnológico (CNPq); Estação Científica Ferreira Penna (ECFPn/MPEG) for the logistical support; Wolmar B. Wosiacki and Izaura Maschio (MPEG) for curatorial assistance. Authors were supported by the CNPq: BSP (Programa de Capacitação Institucional grant number 300138/2017-2), DTFH (grant number 140452/2012-5), GMD (Programa de Capacitação Institucional grant number 300066/2016-3), and LFAM (grant number 305017/20160); CAPES: TMSF (grant number 23038.042984/200830), and LFAM (grant number 88881.119097/2016-01); to CAPES for granting Scholarships (HL and NLB).

\section{REFERENCES}

ALBERT, J. S., P. PETRY \& R. E. REIS, 2011. Major biogeographic and phylogenetic patterns. In: J. S. ALBERT \& R. E. REIS (Ed.): Historical biogeography of neotropical freshwater fishes: 2158. University of California Press, California. DOI: <http://dx.doi. org/10.1525/california/9780520268685.003.0002>.

BARROS, D. F., J. ZUANON, F. P. MENDONÇA, H. M. V. ESPÍRITO-SANTO, A. V. GALUCH \& A. L. M. ALBERNAZ, 2011. The fish fauna of streams in the Madeira-Purus interfluvial region, Brazilian Amazon. Check List 7(6): 768-773. DOI: <http://dx.doi. org/10.15560/11022>.

BEHLING, H. \& M. L. COSTA, 2000. Holocene environmental changes from the Rio Curuá record in the Caxiuanã region, Eastern Amazon Basin. Quaternary Research 53(3): 369-377. DOI: $<$ http://dx.doi.org/10.1006/qres.1999.2117>.

BENONE, N. L., R. LIGEIRO, L. JUEN \& L. F. A. MONTAG, 2018. Role of environmental and spatial processes structuring fish assemblages in streams of the Eastern Amazon. Marine and Freshwater Research 69(2): 243-252. DOI: <https://doi. org/10.1071/MF17103>
BRASIL, 2011. SNUC - Sistema Nacional de Unidades de Conservação da Natureza: Lei n 9.985, de 18 de julho de 2000; Decreto $n^{\circ} 4.340$, de 22 de agosto de 2002; Decreto $n^{\circ}$ 5.746, de 5 de abril de 2006. Plano Estratégico Nacional de Áreas Protegidas: Decreto $n^{\circ}$ 5.758, de 13 de abril de 2006/Ministério do Meio Ambiente: 1-76. MMA/SBF, Brasília.

BRASIL, 2012. Ministério da Pesca e Aquicultura. Instrução Normativa Interministerial $N^{\circ} 001$, de 3 de janeiro de 2012. Available at: <http://www.icmbio.gov.br/cepsul/images/stories/ legislacao/Instrucao_normativa/2012/in_inter_mpa_mma_01_2012 exploracaopeixesnativosexoticosaguascontinentais.pdf $>$. Accessed on: July 25, 2017.

BRASIL, 2014a. Ministério do Meio Ambiente. Portaria n 445, de 17 dezembro de 2014. Diário Oficial da União, 18 dez. 2014. Available at: <http://www.icmbio.gov.br/cepsul/images/stories/legislacao/ Portaria/2014/p_mma_445_2014_lista_peixes_amea\%C3\%A7ados extin\%C3\%A7\%\%C3\%A3o.pdf>. . Accessed on: July 25, 2017.

BRASIL, 2014b. Ministério do Meio Ambiente. Portaria n 467, de 29 de dezembro de 2014. Diário Oficial da União, 30 dez. 2014. Available at: <http://www.florestal.gov.br/documentos/concessoesflorestais/concessoes-florestais-florestas-sob-concessao/flona-decaxiuana/legislacao-aplicacao-edital-de-caxiuana/1462-portariamma-n-467/file>. Accessed on: July 25, 2017.

CASTELLO, L., D. G. MCGRATH, L. L. HESS, M. T. COE, P. A. LEFEBVRE, P. PETRY, M. N. MACEDO, V. F. RENÓ \& C. C. ARANTES, 2013. The vulnerability of Amazon freshwater ecosystems. Conservation Letters 6(4): 217-229. DOI: <http://dx.doi.org/ 10.1111/conl.12008>.

CLARO-GARCÍA, A., L. J. S. VIEIRA, R. B. JARDULI, V. P. ABRAHÃO \& O. A. SHIBATTA, 2013. Fishes (Osteichthyes: Actinopterygii) from igarapés of the rio Acre basin, Brazilian Amazon. Check List 9(6): 1410-1438. DOI: <http://dx.doi.org/10.15560/9.6.1410>.

COSTA, M. L., D. C. KERN, H. BEHLING \& M. S. BORGE, 2002. Geologia. In: P. L. B. LISBOA (Ed.): Caxiuanã: populações tradicionais, meio físico e diversidade biológica: 179-205. Museu Paraense Emílio Goeldi, Belém.

COX-FERNANDES, C., J. PODOS \& J. G. LUNDBERG, 2004. Amazonian ecology: tributaries enhance the diversity of electric fishes. Science 305(5692): 1960-1962. DOI: <http://dx.doi.org/10.1126/ science. $1101240>$.

DIAS, M. S., W. E. MAGNUSSON \& J. ZUANON, 2010. Effects of reduced- impact logging on fish assemblages in Central Amazonia. Conservation Biology 24(1): 278-286. DOI: <http://dx.doi.org/ 10.1111/j.1523-1739.2009.01299.x>.

ESCHMEYER, W. N., R. FRICKE \& R. VAN DER LAAN, 2017. Catalog of fishes: genera, species, references. Institute for Biodiversity Science and Sustainability, San Francisco. Available at: <http://researcharchive.calacademy.org/research/ichthyology/ catalog/fishcatmain.asp >. Accessed on: July 25, 2017. 
FREITAS, T. M. S., V. H. C. ALMEIDA, L. F. A. MONTAG, R. M. ROCHA \& N. F. FONTOURA, 2011a. Seasonal changes in the gonadossomatic index, allometric condition factor and sex ratio of an auchenipterid catfish from Eastern Amazonia. Neotropical Ichthyology 9(4): 839-847. DOI: <http://dx.doi.org/10.1590/ S1679-62252011005000044>.

FREITAS, T. M. S., V. H. C. ALMEIDA, R. M. VALENTE \& L. F. A. MONTAG, 2011b. Feeding ecology of Auchenipterichthys longimanus (Siluriformes: Auchenipteridae) in a riparian flooded forest of Eastern Amazonia, Brazil. Neotropical Ichthyology 9(3): 629-636. DOI: <http://dx.doi.org/10.1590/S167962252011005000032>.

FREITAS, T. M. S., B. S. PRUDENTE, N. F. FONTOURA \& L. F. A. MONTAG, 2014. Length-weight relationships of dominant fish species from Caxiuanã National Forest, Eastern Amazon, Brazil. Journal of Applied Ichthyology 30(5): 1081-1083. DOI: <http:// dx.doi.org/10.1111/jai.12436>.

FREITAS, T. M. S., V. H. C. ALMEIDA, L. F. A. MONTAG \& N. F. FONTOURA, 2016. Predicting size at first sexual maturity from length/weight relationship: a case study with an Amazonian catfish. Neotropical Ichthyology 14(4): e150152. DOI: <http://dx.doi. org/10.1590/1982-0224-20150152>

FREITAS, T. M. S., L. F. A. MONTAG \& R. B. BARTHEM, 2017. Distribution, feeding and ecomorphology of four species of Auchenipteridae (Teleostei: Siluriformes) in Eastern Amazonia, Brazil. Iheringia. Série Zoologia 107: e2017008. DOI: <https:// dx.doi.org/10.1590/1678-4766e2017008>.

HASHIGUTI, D. T. F., R. M. ROCHA \& L. F. A. MONTAG, 2017. Reproductive seasonality of the detritivorous fish Cyphocharax abramoides (Kner, 1958) (Characiformes: Curimatidae) in flooded rivers of the Eastern Amazon. Environmental Biology of Fishes 100(9): 1033-1046. DOI: <http://dx.doi.org/10.1007/s10641-017-0609-y>.

HURD, L. E., R. G. C. SOUSA, F. K. SIQUEIRA-SOUZA, G. J. COOPER, J. R. KAHNE \& C. E. C. FREITAS, 2016. Amazon floodplain fish communities: habitat connectivity and conservation in a rapidly deteriorating environment. Biological Conservation 195: 118-127. DOI: <http://dx.doi.org/10.1016/j.biocon.2016.01.005>.

INSTITUTO CHICO MENDES DE CONSERVAÇÃO DA BIODIVERSIDADE (ICMBIO), 2013. Portaria n 141, de 14 de janeiro de 2013. Aprova o Plano de Manejo da Floresta Nacional de Caxiuanã, localizada no estado do Pará. Diário Oficial da União, 15 jan. 2013. Available at: < http://www.icmbio.gov.br/portal/images/ stories/imgs-unidades-coservacao/portarias/2013/Port_141.pdf $>$. Accessed on: July 25, 2017.

INTERNATIONAL UNION FOR CONSERVATION OF NATURE AND NATURAL RESOURCES (IUCN), 2017. The IUCN Red List of Threatened Species. Available at: <http://www.iucnredlist. org >. Accessed on: July 25, 2017.
JUNK, W. J., M. G. M. SOARES \& P. B. BAYLEY, 2007. Freshwater fishes of the Amazon River basin: their biodiversity, fisheries, and habitats. Aquatic Ecosystem Health \& Management 10(2): $153-$ 173. DOI: <http://dx.doi.org/10.1080/14634980701351023>.

KULLANDER, S. O., 2003. Family Cichlidae. In: R. E. REIS, S. O. KULLANDER \& C. J. FERRARIS JR. (Ed.): Check list of the freshwater fishes of South and Central America: 605-654. EDIPUCRS, Porto Alegre.

LISBOA, P. L. B., 1997. Caxiuanã: 1-446. Museu Paraense Emílio Goeldi, Belém.

LISBOA, P. L. B., M. G. F. BEZERRA \& A. L. R. CARDOSO (Ed.), 2013. Caxiuanã: história natural e ecologia de uma floresta nacional da Amazônia: 1-300. Museu Paraense Emílio Goeldi, Belém.

LOWE-MCCONNELL, R. H., 1999. Estudos ecológicos de comunidades de peixes tropicais: 1-534. EDUSP, São Paulo.

MELO, B. F., R. C. BENINE, R. BRITZKE, C. S. GAMA \& C. OLIVEIRA, 2016. An inventory of coastal freshwater fishes from Amapá highlighting the occurrence of eight new records for Brazil. ZooKeys 606: 127-140. DOI: <http://dx.doi.org/10.3897/ zookeys.606.9297>

MONTAG, L. F. A., T. M. S. FREITAS, W. B. WOSIAKI \& R. B. BARTHEM, 2008. Os peixes da Floresta Nacional de Caxiuanã (municípios de Melgaço e Portel, Pará - Brasil). Boletim do Museu Paraense Emílio Goeldi. Ciências Naturais 3(1): 11-34.

MONTAG, L. F. A., T. M. S. FREITAS, N. L. BENONE, C. P. FERREIRA, W. B. WOSIACKI \& R. B. BARTHEM, 2013. Ecologia de peixes em um "quase-lago" da Amazônia Oriental. In: P. L. B. LISBOA (Ed.): Caxiuanã: paraíso ainda preservado: 577-593. Museu Paraense Emílio Goeldi, Belém.

OLIVEIRA, V. A., M. A. FERREIRA, R. M. ROCHA \& L. F. A. MONTAG, 2017. Reproduction of the duck catfish Ageneiosus ucayalensis in a ria river system. Journal of Fish Biology 90(3): 1062-1069. DOI: <http://dx.doi.org/10.1111/jfb.13209>.

OTA, R. B., H. J. MESSAGE, W. J. GRAÇA \& C. S. PAVANELLI, 2015. Neotropical Siluriformes as a model for insights on determining biodiversity of animal groups. PLoS One 10(7): e0132913. DOI: <http://dx.doi.org/10.1371/journal.pone.0132913>.

PARÁ, 2007. Conselho Estadual de Meio Ambiente. Resolução n 54 , October 24. Avaiable at: <https://www.sema.pa.gov. br/2007/10/24/10059/>. Accessed on: July 25, 2017.

PEIXOTO, L. A. W., G. M. DUTRA, C. D. SANTANA \& W. B. WOSIACKI, 2013. A new species of the electric fish genus Hypopygus (Gymnotiformes: Hypopomidae) from the Lower Amazon Basin, Brazil. Copeia 2013(2): 232-237. DOI: <http:// doi.org/10.1643/CI-12-087> . 
PEIXOTO, L. A. W., G. M. DUTRA \& W. B. WOSIACKI, 2015. The electric glass knifefishes of the Eigenmannia trilineata species-group (Gymnotiformes: Sternopygidae): monophyly and description of seven new species. Zoological Journal of the Linnean Society 175(2): 384-414. DOI: <http://dx.doi.org/10.1111/zoj.12274>.

PRUDENTE, B. S., M. A. P. FERREIRA, R. M. ROCHA \& L. F. A. MONTAG, 2015. Reproductive biology of the piranha Serrasalmus gouldingi (Fink and Machado-Allison 1992) (Characiformes: Serrasalmidae) in "drowned" rivers of the Eastern Amazon. Environmental Biology of Fishes 98(1): 11-22. DOI: <https://doi. org/10.1007/s10641-014-0232-0>.

PRUDENTE, B. S., P. CARNEIRO-MARINHO, R. M. VALENTE \& L. F. A. MONTAG, 2016. Feeding ecology of Serrasalmus gouldingi (Characiformes: Serrasalmidae) in the lower Anapu River region, Eastern Amazon, Brazil. Acta Amazonica 46(3): 259-270. DOI: <http://dx.doi.org/10.1590/1809-4392201600123>.

PRUDENTE, B. S., P. S. POMPEU, L. JUEN \& L. F. A. MONTAG, 2017. Effects of reduced-impact logging on physical habitat and fish assemblages in streams of Eastern Amazonia. Freshwater Biology 62(2): 303-316. DOI: <http://dx.doi.org/10.1111/fwb.12868>.

REIS, R. E., S. O. KULLANDER \& C. J. FERRARIS JR., 2003. Check list of the freshwater fishes of South and Central America: 1-729. EDIPUCRS, Porto Alegre.

REIS, R. E., J. S. ALBERT, F. DI DARIO, M. M. MINCARONE, P. PETRY \& L. A. ROCHA, 2016. Fish biodiversity and conservation in South America. Journal of Fish Biology 89(1): 12-47. DOI: <http:// dx.doi.org/10.1111/jfb.13016>.

SARMENTO-SOARES, L. M. \& R. F. MARTINS-PINHEIRO, 2008. A systematic revision of Tatia (Siluriformes: Auchenipteridae: Centromochlinae). Neotropical Ichthyology 6(3): 495-542. DOI: <http://dx.doi.org/10.1590/S1679-62252008000300022>.
SILVA-OLIVEIRA, C., A. L. C. CANTO \& F. R. V. RIBEIRO, 2016. Stream ichthyofauna of the Tapajós National Forest, Pará, Brazil. ZooKeys 580: 125-144. DOI: <http://dx.doi.org/10.3897/ zookeys.580.6659>.

SILVEIRA, L. F., B. M. BEISIEGEL, F. F. CURCIO, P. H. VALDUJO, M. DIXO, V. K. VERDADE, G. M. T. MATTOX \& P. T. M. CUNNINGHAM, 2010. Para que servem os inventários de fauna? Estudos Avançados 24(68): 173-207. DOI: < http://dx.doi. org/10.1590/S0103-40142010000100015>.

SOUSA, H. L., 2014. Efeito da alteração do hábitat sobre assembleias de peixes em igarapés afogados da Amazônia Oriental. Dissertação (Mestrado em Ecologia Aquática e Pesca) Universidade Federal do Pará, Belém.

WiNEMILLER, K. O., P. B. MCINTYRE, L. CASTELLO, E. FLUET-CHOUINARD, T. GIARRIZZO, S. NAM, I. G. BAIRD, W. DARWALL, N. K. LUJAN, I. HARRISON, M. L. J. STIASSNY, R. A. M. SILVANO, D. B. FITZGERALD, F. M. PELICICE, A. A. AGOSTINHO, L. C. GOMES, J. S. ALBERT, E. BARAN, M. PETRERE JR., C. ZARFL, M. MULLIGAN, J. P. SULLIVAN, C. C. ARANTES, L. M. SOUSA, A. A. KONING, D. J. HOEINGHAUS, M. SABAJ, J. G. LUNDBERG, J. ARMBRUSTER, M. L. THIEME, P. PETRY, J. ZUANON, G. TORRENTE-VILARA, J. SNOEKS, C. OU, W. RAINBOTH, C. S. PAVANELLI, A. AKAMA, A. VAN SOESBERGEN \& L. SÁENZ, 2016. Balancing hydropower and biodiversity in the Amazon, Congo, and Mekong. Science 351(6269): 128-129. DOI: <http://dx.doi.org/10.1126/science. aac7082>. 
Appendix 1. List of fish species collected in different aquatic environment of the Caxiuanã National Forest, Eastern Amazon, Brazil, during the years 1993 to 2012. Legends: ${ }^{a}=$ new record of species coming from voucher checking; ${ }^{b}=$ new record of species from sampling carried out after $2004 ;{ }^{c}=$ species considered for ornamental exploitation; ${ }^{*}=$ no habitat information.

(Continue)

\begin{tabular}{|c|c|c|c|}
\hline \multirow{2}{*}{ Taxon } & \multicolumn{3}{|c|}{ Environment } \\
\hline & River/bay & Stream & Floating bank \\
\hline \multicolumn{4}{|l|}{ MYLIOBATIFORMES } \\
\hline \multicolumn{4}{|l|}{ Potamotrygonidae } \\
\hline Potamotrygon constellata (Vaillant, 1880) & $x$ & & \\
\hline \multicolumn{4}{|l|}{ LEPIDOSIRENIFORMES } \\
\hline \multicolumn{4}{|l|}{ Lepidosirenidae } \\
\hline Lepidosiren paradoxa Fitzinger, 1837 & $x$ & & $x$ \\
\hline \multicolumn{4}{|l|}{ OSTEOGLOSSIFORMES } \\
\hline \multicolumn{4}{|l|}{ Arapaimidae } \\
\hline Arapaima gigas (Schinz, 1822) & $x$ & & \\
\hline \multicolumn{4}{|l|}{ Osteoglossidae } \\
\hline Osteoglossum bicirrhosum (Cuvier, 1829) & $x$ & & \\
\hline \multicolumn{4}{|l|}{ CLUPEIFORMES } \\
\hline \multicolumn{4}{|l|}{ Engraulidae } \\
\hline Anchovia surinamensis (Bleeker, 1865) & $x$ & & \\
\hline Anchoviella cayennensis (Puyo, 1946) ${ }^{b}$ & $x$ & & \\
\hline Lycengraulis batesii (Günther, 1868) & $x$ & & \\
\hline \multicolumn{4}{|l|}{ Pristigasteridae } \\
\hline Ilisha amazonica (Miranda Ribeiro, 1920) & $x$ & & \\
\hline Pellona castelnaeana Valenciennes, 1847 & $x$ & & \\
\hline Pellona flavipinnis (Valenciennes, 1837) & $x$ & & \\
\hline \multicolumn{4}{|l|}{ CHARACIFORMES } \\
\hline \multicolumn{4}{|l|}{ Acestrorhynchidae } \\
\hline Acestrorhynchus falcatus (Bloch, 1794)c & $x$ & & \\
\hline Acestrorhynchus falcirostris (Cuvier, 1819)c & $x$ & & \\
\hline Acestrorhynchus microlepis (Jardine, 1841) & $x$ & $x$ & \\
\hline Gnathocharax steindachneri Fowler, 1913 & & $x$ & \\
\hline Heterocharax virgulatus Toledo-Piza, 2000 & $x$ & & \\
\hline \multicolumn{4}{|l|}{ Anostomidae } \\
\hline Laemolyta proxima (Garman, 1890) & $x$ & & $x$ \\
\hline Leporinus agassizi Steindachner, $1876^{c}$ & $x$ & & $x$ \\
\hline Leporinus fasciatus (Bloch, 1794)c & $x$ & & \\
\hline Leporinus moralesi Fowler, 1942a & & & $x$ \\
\hline Leporinus parae Eigenmann, 1907 & $x$ & & \\
\hline Leporinus trifasciatus Steindachner, 1876 & $x$ & & \\
\hline
\end{tabular}


Appendix 1.

(Continue)

\begin{tabular}{|c|c|c|c|}
\hline \multirow{2}{*}{ Taxon } & \multicolumn{3}{|c|}{ Environment } \\
\hline & River/bay & Stream & Floating bank \\
\hline Schizodon vittatus (Valenciennes, 1850) & $x$ & & \\
\hline \multicolumn{4}{|l|}{ Chalceidae } \\
\hline Chalceus epakros Zanata \& Toledo-Piza, 2004 & $x$ & & \\
\hline \multicolumn{4}{|l|}{ Characidae } \\
\hline Charax pauciradiatus (Günther, 1864) & $x$ & & \\
\hline Hemigrammus bellottii Steindachner, $1882^{c}$ & & $x$ & \\
\hline Hemigrammus levis Durbin, $1908^{c}$ & & & $x$ \\
\hline Hemigrammus ocellifer (Steindachner, 1882) ${ }^{c}$ & & $x$ & $x$ \\
\hline Hemigrammus schmardae (Steindachner, 1882) $)^{\mathrm{b}, \mathrm{c}}$ & & $x$ & $x$ \\
\hline Hemigrammus unilineatus (Gill, 1858), c & & $x$ & \\
\hline Hyphessobrycon bentosi Durbin, $1908^{\mathrm{b}, \mathrm{c}}$ & & $x$ & \\
\hline Hyphessobrycon copelandi Durbin, 1908c & & $x$ & $x$ \\
\hline \multicolumn{4}{|l|}{ Hyphessobrycon diancistrus Weitzman, 1977*, b, c } \\
\hline Hyphessobrycon heterorhabdus (Ulrey, 1894)b,c & & $x$ & \\
\hline Hyphessobrycon aff. moniliger Moreira, Lima \& Costa, 2002 & & $x$ & \\
\hline Hyphessobrycon sp. ${ }^{\text {a }}$ & & $x$ & \\
\hline Jupiaba anteroides (Géry, 1965) b & $x$ & & \\
\hline \multicolumn{4}{|l|}{ Moenkhausia ceros Eigenmann, 1908*, b } \\
\hline Moenkhausia collettii (Steindachner, 1882) ${ }^{c}$ & & $x$ & \\
\hline Moenkhausia lata Eigenmann, $1908^{\mathrm{a}, \mathrm{c}}$ & & $x$ & $x$ \\
\hline Moenkhausia mikia Marinho \& Langeani, 2010a & $x$ & & \\
\hline Priocharax sp. ${ }^{b}$ & & $x$ & \\
\hline Pristella maxillaris (Ulrey, 1894) & & $x$ & \\
\hline Roeboides descalvadensis Fowler, $1932^{\mathrm{a}, \mathrm{c}}$ & $x$ & & \\
\hline Tetragonopterus chalceus Spix \& Agassiz, 1829a, c & $x$ & & \\
\hline Thayeria obliqua Eigenmann, 1908a, c & $x$ & $x$ & \\
\hline \multicolumn{4}{|l|}{ Crenuchidae } \\
\hline Crenuchus spilurus Günther, $1863^{c}$ & $x$ & $x$ & $x$ \\
\hline Microcharacidium eleotrioides (Géry, 1960)a & $x$ & $x$ & \\
\hline \multicolumn{4}{|l|}{ Ctenoluciidae } \\
\hline Boulengerella maculata (Valenciennes, 1850) ${ }^{c}$ & $x$ & & \\
\hline \multicolumn{4}{|l|}{ Curimatidae } \\
\hline Curimata inornata Vari, 1989 & $x$ & & \\
\hline Curimata vittata (Kner, 1858)c & $x$ & & \\
\hline Curimatopsis aff. evelynae Géry, 1964a & & $x$ & \\
\hline Cyphocharax abramoides (Kner, 1858) & $x$ & & \\
\hline
\end{tabular}


Appendix 1.

(Continue)

\begin{tabular}{|c|c|c|c|}
\hline \multirow{2}{*}{ Taxon } & \multicolumn{3}{|c|}{ Environment } \\
\hline & River/bay & Stream & Floating bank \\
\hline Cyphocharax festivus Vari, $1992^{\mathrm{a}}$ & & $x$ & \\
\hline Cyphocharax multilineatus (Myers, 1927) & $x$ & & \\
\hline \multicolumn{4}{|l|}{ Cynodontidae } \\
\hline Cynodon septenarius Toledo-Piza, 2000 & $x$ & & \\
\hline \multicolumn{4}{|l|}{ Erythrinidae } \\
\hline Erythrinus erythrinus (Bloch \& Schneider, 1801) & $x$ & $x$ & \\
\hline Hoplerythrinus unitaeniatus (Spix \& Agassiz, 1829) & & $x$ & \\
\hline Hoplias malabaricus (Bloch, 1794) & $x$ & $x$ & $x$ \\
\hline \multicolumn{4}{|l|}{ Gasteropelecidae } \\
\hline Carnegiella strigata (Günther, 1864) & $x$ & $x$ & \\
\hline \multicolumn{4}{|l|}{ Hemiodontidae } \\
\hline Hemiodus atranalis (Fowler, 1940) & $x$ & & \\
\hline Hemiodus unimaculatus (Bloch, 1794)c & $x$ & & \\
\hline Micromischodus sugillatus Roberts, 1971 & $x$ & & \\
\hline \multicolumn{4}{|l|}{ Iguanodectidae } \\
\hline Bryconops gracilis (Eigenmann, 1908)a & & $x$ & \\
\hline Bryconops melanurus (Bloch, 1794)c & $x$ & & \\
\hline Iguanodectes rachovii Regan, 1912a, c & & $x$ & \\
\hline \multicolumn{4}{|l|}{ Lebiasinidae } \\
\hline Copeina guttata (Steindachner, 1876) & & $x$ & \\
\hline Copella arnoldi (Regan, 1912)a, c & & $x$ & \\
\hline Copella callolepis (Regan, 1912) & & $x$ & \\
\hline Nannostomus beckfordi Günther, $1872^{\mathrm{b}, \mathrm{c}}$ & $x$ & & \\
\hline Nannostomus eques Steindachner, $1876^{c}$ & $x$ & $x$ & \\
\hline Nannostomus limatus Weitzman, 1978 & & & $x$ \\
\hline Nannostomus trifasciatus Steindachner, $1876^{\mathrm{b}, \mathrm{c}}$ & & $x$ & \\
\hline Nannostomus unifasciatus Steindachner, 1876 b & $x$ & & \\
\hline Pyrrhulina semifasciata Steindachner, $1876^{\text {b }}$ & & $x$ & \\
\hline Pyrrhulina sp. & & $x$ & \\
\hline \multicolumn{4}{|l|}{ Serrasalmidae } \\
\hline Catoprion mento (Cuvier, 1819) ${ }^{c}$ & $x$ & & \\
\hline Metynnis sp. & $x$ & & \\
\hline Myloplus arnoldi Ahl, 1936 & $x$ & & \\
\hline Myloplus asterias (Müller \& Troschel, 1844) & $x$ & & \\
\hline Myloplus rhomboidalis (Cuvier, 1818) & $x$ & & \\
\hline Pristobrycon striolatus (Steindachner, 1908)c & $x$ & & \\
\hline
\end{tabular}


Appendix 1.

(Continue)

\begin{tabular}{|c|c|c|c|}
\hline \multirow{2}{*}{ Taxon } & \multicolumn{3}{|c|}{ Environment } \\
\hline & River/bay & Stream & Floating bank \\
\hline Pygopristis denticulata (Cuvier, 1819)c & $x$ & & $x$ \\
\hline Serrasalmus altispinis Merckx, Jégu \& Santos, 2000 & $x$ & & \\
\hline Serrasalmus eigenmanni Norman, 1929c & $x$ & & \\
\hline Serrasalmus gouldingi Fink \& Machado-Allison, 1992 & $x$ & & \\
\hline Serrasalmus rhombeus (Linnaeus, 1766) & $x$ & & \\
\hline Serrasalmus sp. ${ }^{\text {a }}$ & $x$ & & \\
\hline \multicolumn{4}{|l|}{ Triportheidae } \\
\hline Agoniates halecinus Müller \& Troschel, 1845 & $x$ & & \\
\hline Triportheus albus Cope, $1872^{c}$ & $x$ & & \\
\hline Triportheus elongatus (Günther, 1864)a & $x$ & & \\
\hline \multicolumn{4}{|l|}{ SILURIFORMES } \\
\hline \multicolumn{4}{|l|}{ Auchenipteridae } \\
\hline Ageneiosus inermis (Linnaeus, 1766) & $x$ & & \\
\hline Ageneiosus lineatus Ribeiro, Rapp Py-Daniel \& Walsh, 2017ª & $x$ & & \\
\hline Ageneiosus ucayalensis Castelnau, 1855 & $x$ & & \\
\hline Auchenipterichthys longimanus (Günther, 1864) & $x$ & & \\
\hline Auchenipterichthys punctatus (Valenciennes, 1840) b & $x$ & & \\
\hline Auchenipterus nuchalis (Spix \& Agassiz, 1829) & $x$ & & \\
\hline Centromochlus heckelii (De Filippi, 1853) & $x$ & & \\
\hline Pseudauchenipterus nodosus (Bloch, 1794) & $x$ & & \\
\hline Tatia caxiuanensis Sarmento-Soares \& Martins-Pinheiro, 2008 & $x$ & $x$ & \\
\hline Tatia intermedia (Steindachner, 1877) ${ }^{c}$ & $x$ & & \\
\hline Tetranematichthys quadrifilis (Kner, 1858) & $x$ & & \\
\hline Tetranematichthys wallacei Vari \& Ferraris, $2006^{c}$ & $x$ & & \\
\hline Trachelyichthys exilis Greenfield \& Glodek, 1977 & $x$ & & \\
\hline Trachelyopterus galeatus (Linnaeus, 1766) ${ }^{c}$ & $x$ & & \\
\hline Trachelyopterus gr. striatulus (Steindachner, 1877)a & $x$ & & \\
\hline \multicolumn{4}{|l|}{ Callichthyidae } \\
\hline Callichthys callichthys Linnaeus, $1758^{c}$ & & $x$ & \\
\hline Megalechis picta (Müller \& Troschel, 1849)a & $x$ & $x$ & $x$ \\
\hline Megalechis thoracata (Valenciennes, 1840)c & $x$ & $x$ & $x$ \\
\hline \multicolumn{4}{|l|}{ Cetopsidae } \\
\hline Cetopsis coecutiens (Lichtenstein, 1819)c & $x$ & & \\
\hline Helogenes marmoratus Günther, $1863^{c}$ & & $x$ & \\
\hline \multicolumn{4}{|l|}{ Doradidae } \\
\hline Acanthodoras cathaphractus Linnaeus, $1758^{c}$ & & $x$ & \\
\hline
\end{tabular}


Appendix 1.

(Continue)

Taxon

Acanthodoras spinosissimus (Eigenmann \& Eigenmann, 1888)

Astrodoras asterifrons (Kner, 1853) ${ }^{c}$

Megalodoras uranoscopus (Eigenmann \& Eigenmann, 1888)

Ossancora asterophysa Birindelli \& Sabaj Pérez, 2011

Physopyxis ananas Sousa \& Rapp Py-Daniel, 2005

Heptapteridae

Gladioglanis conquistador Lundberg, Bornbusch \& Mago-Leccia, 1991ª

Rhamdia muelleri (Günther, 1864) ${ }^{b}$

Loricariidae

Ancistrus sp.

Farlowella amazonum (Günther, 1864)*,a, c

Hypancistrus lunaorum Armbruster, Lujan \& Taphorn, 2007

Hypoptopoma sp.

Limatulichthys griseus (Eigenmann, 1909)a

Loricaria sp. ${ }^{a}$

Loricarichthys acutus (Valenciennes, 1840)

Otocinclus mura Schaefer, $1997^{\mathrm{b}}$

Panaqolus tankei Cramer \& Sousa, 2016

Peckoltia sp. ${ }^{c}$

Pseudacanthicus hystrix (Valenciennes, 1840) ${ }^{a}$

Pterygoplichthys sp. ${ }^{\text {a }}$

Pimelodidae

Brachyplatystoma filamentosum (Lichtenstein, 1819)

Brachyplatystoma vaillantii (Valenciennes, 1840)

Hypophthalmus fimbriatus Kner, 1858

Hypophthalmus marginatus Valenciennes, 1840

Pimelodina flavipinnis Steindachner, 1876a, c

Pimelodus albofasciatus Mees, 1974

Pimelodus ornatus Kner, 1858

Pimelodus sp.

Platynematichthys notatus (Jardine, 1841)

Pseudoplatystoma punctifer (Castelnau, 1855) ${ }^{\mathrm{a}}$

Pseudopimelodidae

Batrochoglanis aff. villosus (Eigenmann, 1912) $)^{\mathrm{a}_{\mathrm{c}} \mathrm{c}}$

Environment

\begin{tabular}{|c|c|c}
\multicolumn{3}{|c}{ Environment } \\
\cline { 2 - 4 } River/bay & Stream & Floating bank \\
\hline$x$ & & \\
\hline$x$ & $x$ & \\
\hline & & $x$ \\
\hline & & \\
\hline
\end{tabular}

\begin{tabular}{|l|l|l}
\hline & $x$ & \\
\hline$x$ & & \\
\hline
\end{tabular}

Trichomycteridae

Ochmacanthus sp. ${ }^{\mathrm{b}}$

\begin{tabular}{|c|c|c|}
\hline$x$ & & $x$ \\
\hline$x$ & & \\
\hline$x$ & & $x$ \\
\hline$x$ & & \\
\hline$x$ & & \\
\hline$x$ & & \\
\hline & $x$ & \\
\hline$x$ & & \\
\hline$x$ & & \\
\hline$x$ & & \\
\hline$x$ & & \\
\hline$x$ & & \\
\hline$x$ & & \\
\hline$x$ & & \\
\hline$x$ & & \\
\hline$x$ & & \\
\hline$x$ & & \\
\hline$x$ & & \\
\hline$x$ & & \\
\hline$x$ & & \\
\hline$x$ & & \\
\hline & $x$ & $x$ \\
\hline & $x$ & \\
\hline
\end{tabular}


Appendix 1.

(Continue)

\begin{tabular}{|c|c|c|c|}
\hline \multirow{2}{*}{ Taxon } & \multicolumn{3}{|c|}{ Environment } \\
\hline & River/bay & Stream & Floating bank \\
\hline Plectrochilus sp. ${ }^{\mathrm{b}}$ & $x$ & & \\
\hline Potamoglanis hasemani (Eigenmann, 1914) & & $x$ & \\
\hline Tridentopsis sp. ${ }^{\mathrm{b}}$ & & $x$ & \\
\hline Vandellia cirrhosa Valenciennes, $1846^{\mathrm{b}}$ & $x$ & & \\
\hline \multicolumn{4}{|l|}{ GYMNOTIFORMES } \\
\hline \multicolumn{4}{|l|}{ Apteronotidae } \\
\hline Apteronotus aff. albifrons (Linnaeus, 1766) ${ }^{\mathrm{a}, \mathrm{c}}$ & $x$ & & \\
\hline Porotergus sp. ${ }^{a}$ & & & $x$ \\
\hline Sternarchogiton sp. ${ }^{a}$ & & & $x$ \\
\hline \multicolumn{4}{|l|}{ Gymnotidae } \\
\hline Electrophorus electricus (Linnaeus, 1766) & $x$ & $x$ & \\
\hline Gymnotus carapo Linnaeus, $1758^{c}$ & & $x$ & $x$ \\
\hline Gymnotus coatesi La Monte, 1935a & & $x$ & \\
\hline Gymnotus coropinae Hoedeman, $1962^{b}$ & & $x$ & \\
\hline \multicolumn{4}{|l|}{ Hypopomidae } \\
\hline Brachyhypopomus brevirostris (Steindachner, 1868) c & & $x$ & $x$ \\
\hline Brachyhypopomus pinnicaudatus (Hopkins, 1991) & & & $x$ \\
\hline Microsternarchus bilineatus Fernández-Yépez, 1968 & & $x$ & $x$ \\
\hline \multicolumn{4}{|l|}{ Rhamphichthyidae } \\
\hline Gymnorhamphichthys rondoni (Miranda Ribeiro, 1920)c & & $x$ & \\
\hline Hypopygus benoneae Peixoto, Dutra, de Santana \& Wosiacki, 2013 & & $x$ & \\
\hline Hypopygus lepturus Hoedeman, $1962^{c}$ & & $x$ & \\
\hline Rhamphichthys marmoratus Castelnau, $1855^{\mathrm{a}, \mathrm{c}}$ & $x$ & & \\
\hline Steatogenys duidae (La Monte, 1929) b, c & & $x$ & \\
\hline \multicolumn{4}{|l|}{ Sternopygidae } \\
\hline Archolaemus blax Korringa, 1970 & $x$ & & \\
\hline Eigenmannia antonioi Peixoto, Dutra \& Wosiacki, 2015a, c & & & $x$ \\
\hline Eigenmannia aff. macrops (Boulenger, 1897)a & & $x$ & \\
\hline Eigenmannia nigra Mago-Leccia, 1994a, c & $x$ & & \\
\hline Sternopygus macrurus (Bloch \& Schneider, 1801) & $x$ & & $x$ \\
\hline Sternopygus xingu Albert \& Fink, 1996 & & & $x$ \\
\hline \multicolumn{4}{|l|}{ CYPRINODONTIFORMES } \\
\hline \multicolumn{4}{|l|}{ Cynolebiidae } \\
\hline Anablepsoides urophthalmus (Günther, 1866) ${ }^{a}$ & & $x$ & $x$ \\
\hline Laimosemion strigatus (Regan, 1912) ${ }^{\mathrm{a}}$ & & $x$ & $x$ \\
\hline
\end{tabular}


Appendix 1.

(Continue)

\begin{tabular}{|c|c|c|c|}
\hline \multirow{2}{*}{ Taxon } & \multicolumn{3}{|c|}{ Environment } \\
\hline & River/bay & Stream & Floating bank \\
\hline \multicolumn{4}{|l|}{ Poecilidae } \\
\hline Fluviphylax palikur Costa \& Le Bail, 1999a, c & & $x$ & $x$ \\
\hline Pamphorichthys cf. araguaiensis Costa, 1991 & $x$ & & \\
\hline \multicolumn{4}{|l|}{ BELONIFORMES } \\
\hline \multicolumn{4}{|l|}{ Belonidae } \\
\hline Potamorrhaphis guianensis (Jardine, 1843)c & $x$ & & \\
\hline Pseudotylosurus angusticeps (Günther, 1866) & $x$ & & \\
\hline Pseudotylosurus microps (Günther, 1866) ${ }^{\mathrm{a}}$ & $x$ & & \\
\hline \multicolumn{4}{|l|}{ PERCIFORMES } \\
\hline \multicolumn{4}{|l|}{ Cichlidae } \\
\hline Acarichthys heckelii (Müller \& Troschel, 1849)c & $x$ & $x$ & \\
\hline Acaronia nassa (Heckel, 1840) & $x$ & $x$ & $x$ \\
\hline Aequidens tetramerus (Heckel, 1840)c & $x$ & $x$ & \\
\hline Apistogramma gr. agassizii (Steindachner, 1875) & & $x$ & $x$ \\
\hline Apistogramma gr. regani Kullander, $1980^{a}$ & & $x$ & \\
\hline Astronotus crassipinnis (Heckel, 1840)c & $x$ & & \\
\hline Biotodoma cupido (Heckel, 1840) & $x$ & & \\
\hline Caquetaia spectabilis (Steindachner, 1875) ${ }^{c}$ & $x$ & & $x$ \\
\hline Chaetobranchus flavescens Heckel, $1840^{\mathrm{b}}$ & $x$ & & \\
\hline Cichla melaniae Kullander \& Ferreira, 2006 & $x$ & & \\
\hline Cichla monoculus Agassiz, 1831 & $x$ & & \\
\hline Cichla temensis Humboldt, $1821^{\text {a }}$ & $x$ & & \\
\hline Crenicichla macrophthalma Heckel, 1840` & $x$ & & \\
\hline Crenicichla regani Ploeg, $1989^{b, c}$ & $x$ & & \\
\hline Crenicichla strigata Günther, $1862^{\circ}$ & $x$ & & \\
\hline Crenicichla sp. $^{\text {a }}$ & $x$ & $x$ & \\
\hline Geophagus proximus (Castelnau, 1855) & $x$ & & \\
\hline Geophagus aff. surinamensis (Bloch, 1791) & $x$ & & \\
\hline Heros efasciatus Heckel, 1840a, c & & & $x$ \\
\hline Heros notatus (Jardine, 1843) ${ }^{c}$ & $x$ & & \\
\hline Hypselecara coryphaenoides (Heckel, 1840) & $x$ & $x$ & \\
\hline 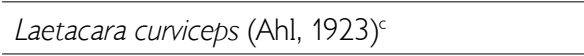 & & $x$ & \\
\hline Mesonauta festivus (Heckel, 1840) & $x$ & $x$ & $x$ \\
\hline Nannacara taenia Regan, 1912 ${ }^{a, c}$ & & $x$ & \\
\hline Pterophyllum scalare (Schultze, 1823)c & & $x$ & \\
\hline
\end{tabular}


Appendix 1.

(Conlusion)

\begin{tabular}{|c|c|c|c|}
\hline \multirow{2}{*}{ Taxon } & \multicolumn{3}{|c|}{ Environment } \\
\hline & River/bay & Stream & Floating bank \\
\hline Satanoperca jurupari (Heckel, 1840)c & $x$ & & \\
\hline Symphysodon aequifasciatus Pellegrin, 1904c & & $x$ & \\
\hline Taeniacara candidi Myers, 1935 & & $x$ & $x$ \\
\hline \multicolumn{4}{|l|}{ Eleotridae } \\
\hline Microphilypnus ternetzi Myers, $1927^{a}$ & $x$ & $x$ & \\
\hline \multicolumn{4}{|l|}{ Polycentridae } \\
\hline Monocirrhus polyacanthus Heckel, $1840^{\circ}$ & & $x$ & \\
\hline Polycentrus schomburgkii Müller \& Troschel, 1849` & & $x$ & \\
\hline \multicolumn{4}{|l|}{ Scianidae } \\
\hline Pachypops fourcroi (Lacepède, 1802) & $x$ & & \\
\hline Plagioscion squamosissimus (Heckel, 1840) & $x$ & & \\
\hline \multicolumn{4}{|l|}{ PLEURONECTIFORMES } \\
\hline \multicolumn{4}{|l|}{ Achiridae } \\
\hline Hypoclinemus mentalis (Günther, 1862) & $x$ & & \\
\hline \multicolumn{4}{|l|}{ TETRAODONTIFORMES } \\
\hline \multicolumn{4}{|l|}{ Tetraodontidae } \\
\hline Colomesus asellus (Müller \& Troschel, 1849)c & $x$ & $x$ & $x$ \\
\hline \multicolumn{4}{|l|}{ SYNBRANCHIFORMES } \\
\hline \multicolumn{4}{|l|}{ Synbranchidae } \\
\hline Synbranchus marmoratus Bloch, 1795 & $x$ & $x$ & $x$ \\
\hline Synbranchus sp. & & & $x$ \\
\hline
\end{tabular}


MYLIOBATIFORMES. Potamotrygonidae. Potamotrygon constellata: MPEG 11711.

OSTEOGLOSSIFORMES. Arapaimidae. Arapaima gigas: MPEG 11302. Osteoglossidae. Osteoglossum bicirrhosum: MPEG 3769, MPEG 11377, MPEG 15280.

CLUPEIFORMES. Engraulidae. Anchovia surinamensis: MPEG 6524, MPEG 8865, MPEG 8952, MPEG 11277, MPEG 11278, MPEG 35290. Anchoviella cayennensis: MPEG 6140. Lycengraulis batesii: MPEG 6499, MPEG 8709, MPEG 9331. Pristigasteridae. Ilisha amazonica: MPEG 8647, MPEG 11306, MPEG 11338, MPEG 27161. Pellona castelnaeana: MPEG 6538, MPEG 11340. Pellona flavipinnis: MPEG 11339.

CHARACIFORMES. Acestrorhynchidae. Acestrorhynchus falcatus: MPEG 11326, MPEG 16213. Acestrorhynchus falcirostris: MPEG 4830, MPEG 19193, MPEG 27166. Acestrorhynchus microlepis: MPEG 4762. Gnathocharax steindachneri: MPEG 6217. Heterocharax virgulatus: MPEG 8176, MPEG 11459. Laemolyta proxima: MPEG 4858, MPEG 8612, MPEG 8681, MPEG 8720, MPEG 8845 , MPEG 18631. Leporinus agassizi: MPEG 8671. Leporinus fasciatus: MPEG 4859, MPEG 8652, MPEG 8698 , MPEG 8701. Leporinus moralesi: MPEG 10187. Leporinus parae: MPEG 8713. Leporinus trifasciatus: MPEG 8690. Schizodon vittatus: MPEG 8992. Chalceidae. Chalceus epakros: MPEG 8638. Characidae. Charax pauciradiatus: MPEG 6525, MPEG 6584, MPEG 8114, MPEG 15447. Hemigrammus bellottii: MPEG 7664. Hemigrammus levis: MPEG 10157, MPEG 10388. Hemigrammus ocellifer: MPEG 11687. Hemigrammus schmardae: MPEG 10302, MPEG 10303. Hemigrammus unilineatus: MPEG 11520, MPEG 11677. Hyphessobrycon aff. moniliger: MPEG 23385. Hyphessobrycon bentosi: MPEG 11264, MPEG 11269. Hyphessobrycon copelandi: MPEG 10147, MPEG 10150.
Hyphessobrycon diancistrus: MPEG 10298. Hyphessobrycon heterorhabdus: MPEG 10391, MPEG 12098, MPEG 12099. Hyphessobrycon sp.: MPEG 9887, MPEG 9968, MPEG 11362. Jupiaba anteroides: MPEG 15445. Moenkhausia ceros: MPEG 11685. Moenkhausia collettii: MPEG 9951, MPEG 10389, MPEG 11254, MPEG 11680. Moenkhausia lata: MPEG 6609, MPEG 6610, MPEG 8600, MPEG 11290. Moenkhausia mikia: MPEG 8731, MPEG 8843, MPEG 8990, MPEG 10134, MPEG 35293, MPEG 35296, MPEG 35299. Priocharax sp.: MPEG 23389. Pristella maxillaris: MPEG 35302. Roeboides descalvadensis: MPEG 8920. Tetragonopterus chalceus: MPEG 8685. Thayeria obliqua: MPEG 6212, MPEG 11450, MPEG 11509, MPEG 11623, MPEG 15517, MPEG 15272. Crenuchidae. Crenuchus spilurus: MPEG 6155, MPEG 10476, MPEG 10484. Microcharacidium eleotrioides: MPEG 6194, MPEG 6561, MPEG 11492, MPEG 11662. Ctenoluciidae. Boulengerella maculata: MPEG 11036, MPEG 19182. Curimatidae. Curimata inornata: MPEG 8570, MPEG 11305, MPEG 11350. Curimata vittata: MPEG 6521, MPEG 11159. Curimatopsis aff. evelynae: MPEG 10142, MPEG 10485, MPEG 11415, MPEG 11447. Cyphocharax abramoides: MPEG 8803, MPEG 8996, MPEG 15539, MPEG 4889. Cyphocharax festivus: MPEG 8750, MPEG 9304, MPEG 15452, MPEG 15518. Cyphocharax multilineatus: MPEG 8700. Cynodontidae. Cynodon septenarius: MPEG 19184, MPEG 8627. Erythrinidae. Erythrinus erythrinus: MPEG 11567, MPEG 11577, MPEG 12023, MPEG 16577, MPEG 16676, MPEG 6512. Hoplerythrinus unitaeniatus: MPEG 23387. Hoplias malabaricus: MPEG 8115, MPEG 8842, MPEG 8910, MPEG 4844. Gasteropelecidae. Carnegiella strigata: MPEG 10410, MPEG 10481, MPEG 11906. MPEG 11908, MPEG 6204. Hemiodontidae. Hemiodus atranalis: MPEG 8863, MPEG 8682, MPEG 8683. Hemiodus unimaculatus: MPEG 15457, MPEG 15460, MPEG 15480, MPEG 18632, MPEG 4972. Micromischodus sugillatus: MPEG 6539, MPEG 11136, MPEG 15469. 
Iguanodectidae. Bryconops gracilis: MPEG 6545, MPEG 6574, MPEG 6582, MPEG 6592, MPEG 8560, MPEG 8617, MPEG 9356, MPEG 35288, MPEG 35294. Bryconops melanurus: MPEG 6543, MPEG 6568, MPEG 6572, MPEG 6575, MPEG 6578, MPEG 6586, MPEG 6587, MPEG 6588, MPEG 6599, MPEG 6602, MPEG 8556, MPEG 8564, MPEG 8571, MPEG 8573, MPEG 8616, MPEG 8661, MPEG 8672, MPEG 8679, MPEG 8684, MPEG 8686, MPEG 8692, MPEG 8727, MPEG 8746, MPEG 8765, MPEG 8771, MPEG 8821, MPEG 8826, MPEG 8829, MPEG 8839, MPEG 8854, MPEG 8862, MPEG 8867, MPEG 8875, MPEG 8908, MPEG 8916, MPEG 8949, MPEG 8950, MPEG 8951, MPEG 8955, MPEG 9309, MPEG 9338, MPEG 9341, MPEG 9342, MPEG 9346, MPEG 9355, MPEG 11621. Iguanodectes rachovi: MPEG 11449. Lebiasinidae. Copeina guttata: MPEG 10415, MPEG 10426, MPEG 10440. Copella arnoldi: MPEG 10416, MPEG 10436, MPEG 11271, MPEG 11491. Copella callolepis: MPEG 10407, MPEG 10437, MPEG 10477, MPEG 11667. Nannostomus beckfordi: MPEG 11276, MPEG 11452, MPEG 11457. Nannostomus eques: MPEG 6114, MPEG 6193, MPEG 6570. Nannostomus limatus: MPEG 6115. Nannostomus trifasciatus: MPEG 6159. Nannostomus unifasciatus: MPEG 11266. Pyrrhulina semifasciata: MPEG 23388. Pyrrhulina sp.: MPEG 10425, MPEG 10480, MPEG 6156. Serrasalmidae. Catoprion mento: MPEG 8770, MPEG 11343, MPEG 18638, MPEG 6583, MPEG 6593. Metynnis sp.: MPEG 19185, MPEG 22938, MPEG 24343, MPEG 6182. Myloplus arnoldi: MPEG 6527, MPEG 11337. Myloplus asterias: MPEG 9362. Myloplus rhomboidalis: MPEG 8781. Pristobrycon striolatus: MPEG 8775, MPEG 8913. Pygopristis denticulata: MPEG 8759, MPEG 8767, MPEG 9322, MPEG 11267, MPEG 19308. Serrasalmus altispinis: MPEG 6505, MPEG 8592, MPEG 8613, MPEG 10019, MPEG 11363, MPEG 19183, MPEG 19318. Serrasalmus eigenmanni: MPEG 8967, MPEG 9590, MPEG 15511. Serrasalmus gouldingi: MPEG 6506, MPEG 6550, MPEG 6603, MPEG 15461, MPEG 15828,
MPEG 35295. Serrasalmus rhombeus: MPEG 11367, MPEG 6505. Serrasalmus sp.: MPEG 6142, MPEG 6507, MPEG 6522, MPEG 8607, MPEG 8607, MPEG 35291, MPEG 8982, MPEG 9322, MPEG 11286. Triportheidae. Agoniates halecinus: MPEG 15471, MPEG 15825, MPEG 18634, MPEG 24337, MPEG 6604. Triportheus albus: MPEG 8611, MPEG 8670, MPEG 8825, MPEG 8864, MPEG 8939, MPEG 11316. Triportheus elongatus: MPEG 6589, MPEG 8744, MPEG 8776.

SILURIFORMES. Auchenipteridae. Ageneiosus inermis: MPEG 8630, MPEG 8719. Ageneiosus lineatus: MPEG 8640, MPEG 8642, MPEG 8643, MPEG 8646, MPEG 8649, MPEG 8651, MPEG 8726, MPEG 8857, MPEG 8877, MPEG 8896, MPEG 11291. Ageneiosus ucayalensis: MPEG 8108, MPEG 8112, MPEG 11715, MPEG 16208. Auchenipterichthys longimanus: MPEG 14851, MPEG 14950, MPEG 15049 , MPEG 15148, MPEG 15247, MPEG 15346, MPEG 15445, MPEG 15544, MPEG 4370. Auchenipterichthys punctatus: MPEG 15838. Auchenipterus nuchalis: MPEG 8724, MPEG 8764, MPEG 8888, MPEG 4357. Centromochlus heckelii: MPEG 8578. Pseudauchenipterus nodosus: MPEG 8582, MPEG 8583. Tatia caxiuanensis: MPEG 6201, MPEG 9859. Tatia intermedia: MPEG 8769, MPEG 8891, MPEG 6601. Tetranematichthys quadrifilis: MPEG 4373, MPEG 6552. Tetranematichthys wallacei: MPEG 6501, MPEG 6528. Trachelyichthys exilis: MPEG 8580, MPEG 8584, MPEG 8666. Trachelyopterus galeatus: MPEG 8804, MPEG 8818, MPEG 10190, MPEG 11349, MPEG 11378, MPEG 35289 , MPEG 35297, MPEG 35298. Trachelyopterus gr. striatulus: MPEG 8664, MPEG 8660, MPEG 8961, MPEG 8986, MPEG 35292. Callichthyidae. Megalechis picta: MPEG 6154, MPEG 9551. Megalechis thoracata: MPEG 6541, MPEG 11387, MPEG 13328. Callichthys callichthys: MPEG 11309, MPEG 11320. Cetpsidae. Cetopsis coecutiens: MPEG 4381, MPEG 8636, MPEG 8659. Helogenes marmoratus: MPEG 11915, MPEG 11916, MPEG 11476. Doradidae.

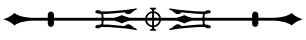


Acanthodoras cathaphractus: MPEG 23392. Acanthodoras spinosissimus: MPEG 11307, MPEG 11529, MPEG 15446, MPEG 16204. Astrodoras asterifrons: MPEG 8645, MPEG 11003, MPEG 15276. Megalodoras uranoscopus: MPEG 11308. Ossancora asterophysa: MPEG 9970, MPEG 10153. Physopyxis ananas: MPEG 6151, MPEG 11919, MPEG 13936. Heptapteridae. Gladioglanis conquistador: MPEG 11655 , MPEG 11663, MPEG 35283. Rhamdia muelleri: MPEG 13408. Loricariidae. Ancistrus sp.: MPEG 11345, MPEG 11816. Farlowella amazonum: MPEG 11602. Hypoptopoma sp.: MPEG 8597, MPEG 10165. Limatulichthys griseus: MPEG 6595, MPEG 6596, MPEG 8730, MPEG 8783. Loricaria sp.: MPEG 8802. Loricarichthys acutus: MPEG 8936, MPEG 8988, MPEG 15278. Otocinclus mura: MPEG 23386. Panaqolus tankei: MPEG 11274. Peckoltia sp.: MPEG 6613, MPEG 6614, MPEG 6615, MPEG 8644, MPEG 8779, MPEG 11292, MPEG 11345, MPEG 11361, MPEG 24346. Pseudacanthicus hystrix: MPEG 9306. Pterygoplichthys sp.: MPEG 6555. Pimelodidae. Brachyplatystoma filamentosum: MPEG 11405. Brachyplatystoma vaillantii: MPEG 11303. Hypophthalmus fimbriatus: MPEG 4377, MPEG 18954. Hypophthalmus marginatus: MPEG 18954 , MPEG 4377. Pimelodina flavipinnis: MPEG 11321. Pimelodus albofasciatus: MPEG 4377. Pimelodus ornatus: MPEG 8844, MPEG 15277. Pimelodus sp.: MPEG 8991, MPEG 11288, MPEG 11348, MPEG 11620. Platynematichthys notatus: MPEG 6560. Pseudoplatystoma punctifer: MPEG 8629. Pseudopimelodidae. Batrochoglanis cf. villosus: MPEG 9980, MPEG 10424. Trichomycteridae. Ochmacanthus sp.: MPEG 10396. Plectrochilus sp.: MPEG 27172, MPEG 27174. Potamoglanis hasemani: MPEG 10395, MPEG 10401, MPEG 10412, MPEG 10499, MPEG 10501, MPEG 12069, MPEG 12071, MPEG 12073, MPEG 12075, MPEG 12077, MPEG 12079, MPEG 12115, MPEG 16560, MPEG 16561, MPEG 16562, MPEG 16563, MPEG 16564, MPEG 16565. Tridentopsis sp.: MPEG 23384. Vandellia cirrhosa: MPEG 27173.
GYMNOTIFORMES. Apteronotidae. Apteronotus aff. albifrons: MPEG 8615, MPEG 11333. Porotergus sp.: MPEG 9886. Sternarchogiton sp.: MPEG 9964. Gymnotidae. Electrophorus electricus: MPEG 6488, MPEG 15270. Gymnotus carapo: MPEG 6490. Gymnotus coatesi: MPEG 11652, MPEG 10173, MPEG 10518, MPEG 23390. Gymnotus coropinae: MPEG 18710, MPEG 18711. Hypopomidae. Brachyhypopomus brevirostris: MPEG 9884. Brachyhypopomus pinnicaudatus: MPEG 3735, MPEG 6482, MPEG 9942, MPEG 9943, MPEG 9962, MPEG 9987, MPEG 10162. Microsternarchus bilineatus: MPEG 6124, MPEG 6483, MPEG 9885, MPEG 10163, MPEG 10510, MPEG 10512, MPEG 10515. Rhamphichthyidae. Gymnorhamphichthys rondoni: MPEG 12041, MPEG 10507. Hypopygus benoneae: MPEG 3729, MPEG 3731, MPEG 18713, MPEG 23603, MPEG 23604, MPEG 23605, MPEG 24295, MPEG 24296. Hypopygus lepturus: MPEG 6210, MPEG 9945, MPEG 9965, MPEG 9967, MPEG 10008, MPEG 10171, MPEG 10509, MPEG 12043, MPEG 12044, MPEG 12045. Rhamphichthys marmoratus: MPEG 8833, MPEG 27171, MPEG 15528, MPEG 6489, MPEG 6491. Steatogenys duidae: MPEG 12042, MPEG 9340. Sternopygidae. Archolaemus blax: MPEG 8596. Eigenmannia aff. macrops: MPEG 8798, MPEG 9319. Eigenmannia antonioi: MPEG 9940, MPEG 10156, MPEG 10167, MPEG 10181, MPEG 10182, MPEG 10186, MPEG 29486, MPEG 11670. Eigenmannia nigra: MPEG 6484, MPEG 8717, MPEG 15522. Sternopygus macrurus: MPEG 6487, MPEG 11323, MPEG 11539. Sternopygus xingu: MPEG 6484, MPEG 8657, MPEG 10015, MPEG 10191.

CYPRINODONTIFORMES. Cynolebiidae. Anablepsoides urophthalmus: MPEG 9946, MPEG 9954, MPEG 11615, MPEG 11650. Laimosemion strigatus: MPEG 9949, MPEG 9984, MPEG 10154, MPEG 10434, MPEG 11584. Poecilidae. Fluviphylax palikur: MPEG 11574, MPEG 11581,

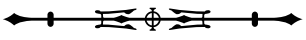


MPEG 11603, MPEG 11611, MPEG 11613, MPEG 11661. Pamphorichthys cf. araguaiensis: MPEG 6564.

BELONIFORMES. Belonidae. Potamorrhaphis guianensis: MPEG 6203, MPEG 11330, MPEG 11702. Pseudotylosurus angusticeps: MPEG 8780, MPEG 8929. Pseudotylosurus microps: MPEG 11708.

PERCIFORMES. Cichlidae. Acarichthys heckelii: MPEG 4916, MPEG 8662, MPEG 11409, MPEG 11601. Acaronia nassa: MPEG 3835, MPEG 9875, MPEG 10141, MPEG 11282, MPEG 11335. Aequidens tetramerus: MPEG 6446, MPEG 6450, MPEG 6472, MPEG 10505, MPEG 23438, MPEG 27160. Apistogramma gr. agassizi: MPEG 6117, MPEG 10482. Apistogramma gr. regani: MPEG 9881, MPEG 10137, MPEG 10139, MPEG 23391. Astronotus crassipinnis: MPEG 11402. Caquetaia spectabilis: MPEG 10519, MPEG 6454. Biotodoma cupido: MPEG 6131. Chaetobranchus flavescens: MPEG 6476. Cichla melaniae: MPEG 6478. Cichla monoculus: MPEG 11391, MPEG 15294, MPEG 6477. Cichla temensis: MPEG 8831, MPEG 11395, MPEG 3840. Crenicichla macrophthalma: MPEG 9329. Crenicichla regani: MPEG 6444. Crenicichla sp.1: MPEG 6479, MPEG 8790, MPEG 10180. Crenicichla strigata: MPEG 3841, MPEG 8609, MPEG 8693, MPEG 8999. Geophagus aff. surinamensis: MPEG 6462, MPEG 15267, MPEG 15269, MPEG 15467, MPEG 6178. Geophagus proximus: MPEG 8563, MPEG 8603, MPEG 8604, MPEG 8641, MPEG 11280. Heros efasciatus: MPEG 6191, MPEG 8752. Heros notatus: MPEG 6166, MPEG 10417. Hypselecara coryphaenoides: MPEG 9909, MPEG 6447. Laetacara curviceps: MPEG 6119, MPEG 6120, MPEG 6452, MPEG 10138, MPEG 10161. Mesonauta festivus: MPEG 6177, MPEG 9908, MPEG 11546. Nannacara taenia: MPEG 6150, MPEG 6445, MPEG 6467, MPEG 11263. Pterophyllum scalare: MPEG 6453. Satanoperca jurupari: MPEG 6475, MPEG 8118, MPEG 9122, MPEG 6475. Symphysodon aequifasciatus: MPEG 11273, MPEG 11531, MPEG 11373. Taeniacara candidi: MPEG 9883, MPEG 23431. Microphilypnus ternetzi: MPEG 10421, MPEG 11412. Polycentridae. Monocirrhus polyacanthus: MPEG 10488, MPEG 12091. Polycentrus schomburgkii: MPEG 11258. Scianidae. Pachypops fourcroi: MPEG 8614, MPEG 6138. Plagioscion squamosissimus: MPEG 3808, MPEG 8677, MPEG 8721, MPEG 8801.

PLEURONECTIFORMES. Achiridae. Hypoclinemus mentalis: MPEG 6139, MPEG 8655, MPEG 8806.

TETRAODONTIFORMES. Tetraodontidae. Colomesus asellus: MPEG 6134, MPEG 8121, MPEG 9960, MPEG 9972, MPEG 11518.

SYNBRANCHIFORMES. Synbranchidae. Synbranchus marmoratus: MPEG 9955, MPEG 10192. Synbranchus sp.: MPEG 9939, MPEG 9977, MPEG 11582, MPEG 14942, MPEG 11525.

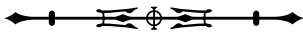

